\title{
New records of the millipede genus Schizoturanius Verhoeff, 1931 from Asian Russia (Diplopoda: Polydesmida: Polydesmidae)
}

\section{Новые находки двупарноногих многоножек рода Schizoturanius Verhoeff, 1931 из азиатской части России (Diplopoda: Polydesmida: Polydesmidae)}

\author{
P.S. Nefediev \\ П.С. Нефедьев
}

Altai State University, Lenin Avenue 61, Barnaul 656049, Russia. E-mail: p.nefediev@mail.ru
Tomsk State University, Lenin Avenue 36, Tomsk 634050, Russia.
Tigirek State Nature Reserve, Nikitina Street 111, Barnaul 656049, Russia.
Алтайский государственный университет, проспект Ленина 61, Барнаул 656049, Россия.
Томский государственный университет, проспект Ленина 36, Томск 634050, Россия.
Государственный природный заповедник «Тигирекский», улица Никитина 111, Барнаул 656049, Россия.

KEY WORDS: Schizoturanius, dmitriewi, clavatipes, tabescens, fauna, new records, Siberia, Asian Russia.

КЛЮЧЕВЫЕ СЛОВА: Schizoturanius, dmitriewi, clavatipes, tabescens, фауна, новые находки, Сибирь, азиатская Россия.

ABSTRACT. Detailed records of Schizoturanius dmitriewi (Timotheew, 1897) from the Asian part of Russia with new ones from the Republic of Altai, as well as the easternmost records of $S$. tabescens (Stuxberg, 1876) from the Irkutsk Area are provided. The main differences in gonopodal structure between the closely related $S$. dmitriewi and $S$. clavatipes (Stuxberg, 1876) are shown. Spatial relationships between all these three species of Schizoturanius are briefly discussed. The distributions of all Schizoturanius species encountered are mapped. A key to all known species of the genus from Asian Russia is given.

РЕЗЮМЕ. Приведены подробные находки Schizoturanius dmitriewi (Timotheew, 1897) из азиатской части России с новыми данными из Республики Алтай, а также самые восточные находки $S$. tabescens (Stuxberg, 1876) из Иркутской области. Показаны основные отличия строения гоноподов у близкородственных $S$. dmitriewi и S. clavatipes (Stuxberg, 1876). Кратко обсуждены пространственные взаимоотношения, сложившиеся между этими тремя видами Schizoturanius. Для всех включённых в настоящую статью видов Schizoturanius выполнено картирование ареалов. Составлен ключ для всех известных из азиатской части России видов этого рода.

\section{Introduction}

The polydesmid genus Schizoturanius Verhoeff, 1931 is presently known to comprise eight species, almost all being confined to Central Asia [Mikhaljova, 2017]. Only one species, S. dmitriewi (Timotheew, 1897), originally described from eastern Ukraine [Timotheew, 1897], later recorded from much of Ukraine and southern Russia [Lokshina, 1966], considered subendemic to the forested steppe belt of the Russian Plain [Golovatch, 1984, 1992] or strictly endemic to the Plain's areas lying between the Dnepr (= Dnieper) and Don rivers [Wytwer et al., 2009], has recently been found in Asian Russia [Krugova, Nefediev, 2018; Nefediev, Nefedieva, 2018]. Detailed new records of $S$. dmitriewi allow for both the distribution area of this species to be refined and the main differences in its gonopodal structure from congeners to be clarified.

\section{Material and methods}

SEM micrographs were prepared at the Laboratory of Phylogeny and Faunogenesis, Institute of Systematics and Ecology of Animals, Siberian Branch of the Russian Academy of Sciences, Novosibirsk, Russia (ISEA), using a Hitachi TM-1000 scanning electron

How to cite this article: Nefediev P.S. 2019. New records of the millipede genus Schizoturanius Verhoeff, 1931 from Asian Russia (Diplopoda: Polydesmida: Polydesmidae) // Russian Entomol. J. Vol.28. No.3. P.331-339. doi: 10.15298/rusentj.28.3.14 
microscope, as well as at the Laboratory of Aquatic Ecology, Institute for Water and Environmental Problems, Siberian Branch, Russian Academy of Sciences, Barnaul, Russia (IWEP), using a Hitachi S-3400N scanning electron microscope. Mounts for SEM were made through air-drying, mounting on stubs, and coating with platinum (in IWEP only). SEM material was removed from stubs and returned to alcohol after examination. Digital images were prepared with the help of Helicon Focus 6 image stacking software. The distribution maps were composed using QGIS 3.6.1Noosa.

The material treated herein has been deposited mainly in the collection of the Altai State University, Barnaul, Russia (ASU), partly shared also with the collection of the Zoological Museum of the Lomonosov Moscow State University, Moscow, Russia (ZMUM). Literature references to the species concern only their distribution areas in the Asian part of Russia.

Gonopod terminology follows that accepted in Golovatch [2014, 2015].

Abbreviations used: s.l. — same locality, s.s. — soil samples, p.t. - pitfall traps, h.s. — hand sampling, TSNR - Tigirek State Nature Reserve, J.N. - J.S. Nefedieva, Kh.N. - Kh.Kh. Nedoev, P.N. - P.S. Nefediev, S.N. - S.T. Niyazov, T.K. - T.M. Krugova, V.S. - V.Yu. Slatina, Yu.D. - Yu.V. Dyachkov (all Barnaul, Russia), V.G. - V.I. Gusarov, M.M. M.F. Maurstad, V.L. - V. Løveng (all Oslo, Norway).

\section{Taxonomic part}

\section{Class Diplopoda}

Family POLYDESMIDAE

\section{Schizoturanius dmitriewi (Timotheew, 1897)}

Figs 1-2, 4, 6, 8.

non Turanodesmus dmitrievi (sic!) - Byzova, Chadaeva, 1965: 337. Schizoturanius clavatipes - Nefediev et al., 2017: 13; 2018: 228 Schizoturanius clavatipes pro parte - Nefediev, Nefedieva, 2017: 292. Schizoturanius dmitriewi - Nefediev, Nefedieva, 2018: 142; Krugova, Nefediev, 2018: 117.

MATERIAL EXAMINED. $1+$ (ASU), Russia, Altai Province, Zmeinogorsk District, TSNR, Beloretsk site, right bank in mouth of Glukharikha River, left bank of Belaya River, $51^{\circ} 00,188 \mathrm{~N}$, $82^{\circ} 45,812^{\prime} \mathrm{E}$, upper part of $\mathrm{W}$ slope, chern taiga, $600 \mathrm{~m}$ a.s.l., p.t., 29.VI.2014; 2 우 (ASU), same locality, p.t., 20-28.VIII.2014; 2 $\mathrm{O}^{7} \mathrm{O}^{\top}$ (ASU), near s.1., $51^{\circ} 00^{\prime} 13.65 \mathrm{~N}, 82^{\circ} 45^{\prime} 46.16^{\prime \prime} \mathrm{E}$, W slope, chern taiga (mainly Abies sibirica), ca. $545 \mathrm{~m}$ a.s.l., p.t., 1328.VIII.2015; $4 \sigma^{7} \sigma^{7}, 1$ (ASU), near s.1., TSNR, buffer zone, $51.000014^{\circ} \mathrm{N}, 82.750219^{\circ} \mathrm{E}$, lower part of W slope, ca. $560 \mathrm{~m}$ a.s.1., p.t., 3-9.VI.2014; 1 क (ASU), s.1., p.t., 20-28.VIII.2014; 1 o (ASU), near s.1., TSNR, Beloretsk site, $51^{\circ} 00,211^{\prime} \mathrm{N}, 82^{\circ} 45,815^{\prime} \mathrm{E}$, upper and middle part of $\mathrm{W}$ slope, ca. $560 \mathrm{~m}$ a.s.1., p.t., 20 28.VIII.2014; 1 (ASU), same Province, Krasnoshchiokovo District, near Tigirek, TSNR, buffer zone, Mt Kozyr, $51^{\circ} 09,135 \mathrm{~N}$, $83^{\circ} 01,196^{\prime} \mathrm{E}$, upper part of steep NE slope, Larix sibirica stand in Larix sibirica and Betula pendula forest, $650 \mathrm{~m}$ a.s.1., p.t., 29.VIII.8.IX.2014; 1 ㅇ (ASU), same Province and District, near Tigirek, TSNR, buffer zone, right bank of Malyi Tigirek River behind ford, valley bottom, $51^{\circ} 08,751 \mathrm{~N}, 83^{\circ} 02,567^{\prime} \mathrm{E}$, valley Betula pendula forest, $480 \mathrm{~m}$ a.s.1., p.t., 29.VIII-8.IX.2014; 1 juv. (ASU), same Province and District, near Tigirek, TSNR, Khankhara site, left bank of Dragunskii Brook, $51^{\circ} 11,502{ }^{\prime} \mathrm{N}, 82^{\circ} 58,778^{\prime} \mathrm{E}$, middle part of S slope, rich herbaceous meadow with Poaceae, ca. $910 \mathrm{~m}$ a.s.1.,

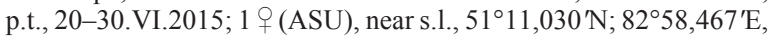
lower part of slope, tall grasses, ca. $800 \mathrm{~m}$ a.s.1.,p.t., 15-25.VIII.2015; 1 ㅇ (ASU), near s.1., $51^{\circ} 11,306^{\prime} \mathrm{N} ; 82^{\circ} 58,569^{\prime} \mathrm{E}$, middle part of slope, rich herbaceous steppe with Carex, Festuca and bushes, ca. $910 \mathrm{~m}$ a.s.1., p.t., 15-25.VIII.2015; 2 우 (ASU), near s.1., $51^{\circ} 11,229^{\prime} \mathrm{N} ; 82^{\circ} 58,546^{\prime} \mathrm{E}$, middle part of slope, meadow steppe with bushes, ca. $875 \mathrm{~m}$ a.s.1., p.t., 15-25.VIII.2015, all leg. T.K.; 2 $\mathrm{O}^{\top} \sigma^{\top}, 1 \stackrel{9}{ }, 1$ juv. (ASU), near s.1., TSNR, buffer zone, $51^{\circ} 11^{\prime} 01.11^{\prime \prime} \mathrm{N}$, $82^{\circ} 58^{\prime} 29.57^{\prime \prime} \mathrm{E}$, tall grasses meadow, bottom of broad gully, $800 \mathrm{~m}$ a.s.1., s.s., 16-17.VIII.2016, leg. T.K., L.Yu. Gruntova, V.V. Zelenskii, K.V. Smirnova, A.E. Pupkova, M.N. Terioshkina, R.V. Shcherbakova; 2 ○ $^{7}, 6$ juv. (ASU), near s.1., SW steppe slope with Rosa spinosissima, h.s., 17.VIII.2016; 1 \%, 1 juv. (ASU), near s.1., Betula pendula stand with Lonicera and Rosa spinosissima, h.s., 17.VIII.2016; 1 ㅇ (ASU), near s.1., $51.192035^{\circ} \mathrm{N}, 82.953123^{\circ} \mathrm{E}$, Betula pendula and Larix sibirica forest, $850 \mathrm{~m}$ a.s.1., h.s., 18.VIII.2016, leg. P.N.; $1 \sigma^{7}, 1$ juv. (ASU), same Province, Charyshskoye District, left bank of Inya River, near Kamyshenka (abandoned), $51^{\circ} 07^{\prime} 04.1^{\prime \prime} \mathrm{N}, 83^{\circ} 06^{\prime} 51.8^{\prime \prime} \mathrm{E}$, chern taiga, $555 \mathrm{~m}$ a.s.1., p.t., 18.VI-3.VII.2016; $1 \mathrm{O}^{7}, 1$ + 4 juv. (ASU), s.1., p.t., 11.VII-3.IX.2016; $1{ }^{\circ}, 2$ juv. (ASU), near s.1., $51^{\circ} 07^{\prime} 00.4^{\prime \prime} \mathrm{N}, 83^{\circ} 06^{\prime} 55.8^{\prime \prime} \mathrm{E}$, chern taiga, $585 \mathrm{~m}$ a.s.1., p.t., 11.VII-3.IX.2016, all leg. T.K; $1 \mathrm{O}^{\text {T }}$ (ASU), same Province and District, ca. $4.5 \mathrm{~km} \mathrm{SE}$ of Charyshskoye, $51^{\circ} 21^{\prime} 33.8^{\prime \prime} \mathrm{N}, 83^{\circ} 37^{\prime} 23.2^{\prime \prime} \mathrm{E}$, Betula pendula and Populus tremula stand on $\mathrm{N}$ slope, ca. $520 \mathrm{~m}$ a.s.1., 5.VI.2018; 4 juv. (ASU), near s.1., site 1 on $\mathrm{S}$ slope, $51^{\circ} 21^{\prime} 20.3^{\prime \prime} \mathrm{N}, 83^{\circ} 37^{\prime} 36.5^{\prime \prime} \mathrm{E}$, s.s. $1(0-10 \mathrm{~cm}$ deep), $480 \mathrm{~m}$ a.s.1., 8.VI.2018; 1 juv. (ASU), s.1., s.s. 1 (10-20 cm deep), 8.VI.2018; 4 juv. (ASU), s.1., s.s. 3 (0-10 cm deep), 8.VI.2018; 7 juv. (ASU), s.1., s.s. 5 (0-10 cm deep), 8.VI.2018; 2 juv. (ASU), s.1., s.s. 5 (10-20 cm deep), 8.VI.2018, all leg. Kh.N., S.N., V.S.; 9 juv. (ASU), s.1., s.s. 3 (0-10 cm deep), 14.VII.2018; 1 juv. (ASU), near s.1., site 2 on S slope, $51^{\circ} 21^{\prime} 14.5^{\prime \prime} \mathrm{N}, 83^{\circ} 38^{\prime} 03.8^{\prime \prime} \mathrm{E}$, s.s. $3(0-10$ $\mathrm{cm}$ deep), $530 \mathrm{~m}$ a.s.1., 13.VII.2018; 1 juv. (ASU), near s.1., site 1 on $\mathrm{N}$ slope, $51^{\circ} 21^{\prime} 44.3^{\prime \prime} \mathrm{N}, 83^{\circ} 37^{\prime} 42.6^{\prime \prime} \mathrm{E}$, s.s. 3 (0-10 cm deep), $620 \mathrm{~m}$ a.s.1., 12.VII.2018; 1 juv. (ASU), near s.1., site 2 on $\mathrm{N}$ slope, $51^{\circ} 21^{\prime} 38.0^{\prime \prime} \mathrm{N}, 83^{\circ} 38^{\prime} 02.7^{\prime \prime} \mathrm{E}$, s.s. $5(0-10 \mathrm{~cm}$ deep), $630 \mathrm{~m}$ a.s.1., 12.VII.2018; 4 O $^{\top}, 3$ 우, 1 juv. (ASU), near s.1., site 1 on S slope, $51^{\circ} 21^{\prime} 20.3^{\prime \prime} \mathrm{N}, 83^{\circ} 37^{\prime} 36.5^{\prime \prime}$, s.s. 1 (0-10 cm deep), $480 \mathrm{~m}$ a.s.l., 6.IX.2018; $1 \mathrm{O}^{7}$ (ASU), s.1., s.s. 1 (10-20 cm deep), 6.IX.2018; $1 \sigma^{7}$, 4 ㅇ (ASU), s.1., s.s. 3 (0-10 cm deep), 6.IX.2018; 1 ○', 2 + (ASU), s.1., s.s. 5 (0-10 cm deep), 6.IX.2018; $1 \sigma^{7}$ (ASU), near s.1., site 1 on $\mathrm{N}$ slope, $51^{\circ} 21^{\prime} 44.3^{\prime \prime} \mathrm{N}, 83^{\circ} 37^{\prime} 42.6^{\prime \prime} \mathrm{E}$, s.s. 1 (0-10 cm deep), $620 \mathrm{~m}$ a.s.1., 7.IX.2018; $2 \sigma^{\top} \sigma^{\top}, 1$ q (ASU), s.1., s.s. 2 (0-10 cm deep), 7.IX.2018; $1 \mathrm{O}^{7}$ (ASU), s.1., s.s. 4 (0-10 cm deep), 7.IX.2018, all leg. Kh.N.; $3 \sigma^{7} \sigma^{7}$ (ASU), Russia, Republic of Altai, Ust-Koksa District, $0.5 \mathrm{~km} \mathrm{~W}$ of Ust-Koksa, near main building of Katun State Nature Reserve, $50^{\circ} 16^{\prime} 27.74^{\prime} \mathrm{N}, 85^{\circ} 35^{\prime} 29.21^{\prime \prime} \mathrm{E}$, Betula pendula forest with Poaceae, in litter and under stones, $980 \mathrm{~m}$ a.s.1., 14 15.VIII.2016, leg. Yu.D.

MATERIAL RE-EXAMINED (specimens recorded by Nefediev, Nefedieva [2017] and Nefediev et al. [2017, 2018]). $1 \sigma^{\top}, 2$, 0,1 fragm. (ASU), Russia, Altai Province, Krasnoshchiokovo District, near Tigirek, TSNR, Khankhara site, $51^{\circ} 11.569$ N, $82^{\circ} 58.740^{\prime} \mathrm{E}$, Larix sibirica forest, 4-year old fire-site, upper part on S slope, left bank of Dragunskii Brook, p.t., 23.V-1.VI.2012; $2 \sigma^{7} \sigma^{7}$ (ASU), same Province, Zmeinogorsk District, TSNR, Beloretsk site, $51^{\circ} 00.277 \mathrm{~N}$, $82^{\circ} 45.830$ 'E, dead-surface Abies sibirica forest (chern taiga), upper part on N slope, 537 m a.s.l., p.t., 28.V.5.VI.2013, all leg. T.K.; 10 juv. (ASU), same Province, Krasnoshchiokovo District, ca. $0.75 \mathrm{~km}$ E of Tigirek, buffer zone of TSNR, $51^{\circ} 08.613 \mathrm{~N}, 83^{\circ} 02.725^{\prime} \mathrm{E}$, Betula forest, $503 \mathrm{~m}$ a.s.1., p.t., 1.VII-6.VII.2013; $2 \sigma^{\top} \sigma^{\top}, 3$ OO, 33 juv., 2 fragm. (ASU), s.1., s.s., 4.VII.2013, all leg. Yu.D.; 2 juv. (ASU), same Province, Charyshskoye District, ca. $4.5 \mathrm{~km}$ SE of Charyshskoye, environs of ASU Field Station "Goluboi Utios", 51²1'33.8'N,

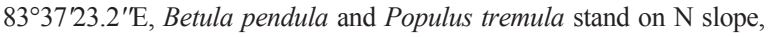
ca. $520 \mathrm{~m}$ a.s.1., 14.VII.2015, leg. P.N.; 4 juv. (ASU), near s.1., site 1 on S slope, $51^{\circ} 21^{\prime} 20.3^{\prime \prime} \mathrm{N}, 83^{\circ} 37^{\prime} 36.5^{\prime \prime} \mathrm{E}$, s.s. 3 (0-10 cm deep), $480 \mathrm{~m}$ a.s.l., 1.VI.2016; 2 +⿱ 9 juv. (ASU), s.1., s.s. 4 (0-10 cm deep), 1.VI.2016; 1 juv. (ASU), s.1., s.s. 5 (0-10 cm deep), 1.VI.2016; $2 \bigcirc^{7} \sigma^{\prime}$, 1 ㅇ, 2 juv. (ASU), near s.1., foot of S slope of mountain, $51.354745^{\circ} \mathrm{N}$, $83.626265^{\circ} \mathrm{E}$, Padus avium and Populus tremula stand near Pikhtovka 
River, h.s., 495 m a.s.l., 1.VI.2016; $20^{7} \sigma^{7}, 2$ 2 (ASU), near s.1., site 1 on $\mathrm{N}$ slope, $51^{\circ} 21^{\prime} 44.3^{\prime \prime} \mathrm{N}, 83^{\circ} 37^{\prime} 42.6^{\prime \prime} \mathrm{E}$, h.s., $620 \mathrm{~m}$ a.s.1., 2.VI.2016, all leg. P.N., Kh.N., S.N., V.S.; 2 juv. (ASU), near s.l., site 1 on S slope, $51^{\circ} 21^{\prime} 20.3^{\prime \prime} \mathrm{N}, 83^{\circ} 3736.5^{\prime \prime}$, s.s. $1(0-10 \mathrm{~cm}$ deep), $480 \mathrm{~m}$ a.s.l. 12.VII.2016; 1 juv. (ASU), s.l., s.s. 3 (0-10 cm deep), 12.VII.2016; 3 juv. (ASU), s.l., s.s. 3 (10-20 cm deep), 12.VII.2016; 2 juv. (ASU), s.1., s.s. 5 (0-10 cm deep), 12.VII.2016; 1 juv. (ASU), near s.l., site 1 on $\mathrm{N}$ slope, $51^{\circ} 21^{\prime} 44.3^{\prime} \mathrm{N}, 83^{\circ} 37^{\prime} 42.6^{\prime \prime} \mathrm{E}$, s.s. $1(0-10 \mathrm{~cm}$ deep), $620 \mathrm{~m}$ a.s.1., 13.VII.2016; 1 juv. (ASU), s.1., h.s., 13.VII.2016; 1 juv. (ASU), near s.l., site 2 on $\mathrm{N}$ slope, $51^{\circ} 21^{\prime} 38.0^{\prime \prime} \mathrm{N}, 83^{\circ} 38^{\prime} 02.7^{\prime \prime} \mathrm{E}$, s.s. $3(0-10 \mathrm{~cm}$ deep), $630 \mathrm{~m}$ a.s.1., 13.VII.2016; 1 juv. (ASU), near s.l., environs of Komendantka, $51.344018^{\circ} \mathrm{N}, 83.593782^{\circ} \mathrm{E}$, h.s., $485 \mathrm{~m}$ a.s.1., 14.VII.2016, all leg. Kh.N., S.N., V.S.; $10^{7}$ (ASU), near s.l., site 1 on S slope, $51^{\circ} 21^{\prime} 20.3^{\prime \prime} \mathrm{N}, 8^{\circ} 37^{\prime} 36.5^{\prime \prime} \mathrm{E}$, s.s. 2 (10-20 cm deep), $480 \mathrm{~m}$ a.s.1.,22.VIII.2016; $1 \mathrm{O}^{\text {, }}$ (ASU), s.1., s.s. 4 (0-10 cm deep), 23.VIII.2016; $1 \mathrm{O}^{7}, 1$ + (ZMMU), 1 ( (ASU), s.1., s.s. 5 (0-10 cm deep), 23.VIII.2016; $1 \mathrm{O}^{\prime \prime}$ (ASU), near s.1., site 2 on S slope, $51^{\circ} 21^{\prime} 14.5^{\prime \prime} \mathrm{N}, 83^{\circ} 38^{\prime} 03.8^{\prime \prime} \mathrm{E}$, s.s. 2 (0-10 cm deep), $530 \mathrm{~m}$ a.s.l., 22.VIII.2016; 1 juv. (ASU), s.1., s.s. 4 (0-10 cm deep), 22.VIII.2016; $1 \mathrm{O}^{7}$ (ASU), near s.l., site 2 on N slope, $51^{\circ} 21^{\prime 3} 38.0^{\prime \prime} \mathrm{N}, 83^{\circ} 38^{\prime} 02.7^{\prime \prime} \mathrm{E}$, s.s. $2(0-10 \mathrm{~cm}$ deep), $630 \mathrm{~m}$ a.s.l., 23.VIII.2016; 1 q (ASU), s.l., s.s. 4 (litter), 23.VIII.2016, all leg. P.N., Kh.N., S.N., V.S.

MATERIAL RE-EXAMINED. $70^{7} O^{7}, 7$ 오 (ZMUM, Rd 1523) Russia, Voronezh Area, Voronezh Nature Reserve, Alnus forest, site 509, 20.IX.1988, leg. V. Emets.

DISTRIBUTION. Until recently, this species was considered to be endemic to the Russian Plain (see above and Zalesskaja et al. [1982]), with the distribution area covering the territory of central and eastern Ukraine and the adjacent regions of Russia east of Ukraine, and further north up to the southern parts of the Moscow Area [Evsyukov, Golovatch, 2013]. Recently, however, this species has been recorded from the Russian Altais generally [Nefediev, Nefedieva, 2018] and from the Tigirek State Nature Reserve in particular [Krugova, Nefediev, 2018], but without precise faunistic data presented (Fig. 1).

REMARKS. Originally described in a new genus, Trachynotus [Timotheew, 1897], this species was later transferred to Schizoturanius [Lohmander, 1933], because the name Trachynotus had been preoccupied. A restudy of the specimens from the south of the Altai Province previously determined by Nefediev, Nefedieva [2017] and Nefediev et al. [2017, 2018] as Schizoturanius clavatipes (Stuxberg, 1876) shows that they actually belong to $S$. dmitriewi.

The species Schizoturanius dmitriewi is very similar to $S$. clavatipes, but differs from it and other species of the genus mainly in the absence of a small triangular blade at the inner edge of the middle part of the gonopod exomere (ex) (Figs 45 ), as well as the serrate/denticulate outer edge of the gonopod endomere (en) (Figs 6-9), in which the distal part is directed caudad, shortened and swollen ventrally, and it carries a well-developed, long and thin process (p) near the $\mathrm{pad} /$ pulvillus (Figs 2-3).

Schizoturanius dmitriewi sometimes occur syntopically with S. tabescens (Stuxberg, 1876) (Figs 1, 12), but the former species is easily distinguished from the latter by the larger body (adults 912 vs. $79 \mathrm{~mm}$ long, respectively) and coloration (pinkish beige $v s$. milky white, respectively). At the same time, S. dmitriewi is quite similar to $S$. clavatipes in habitus, as well as in gonopodal structure, but they never live together, being allopatric.

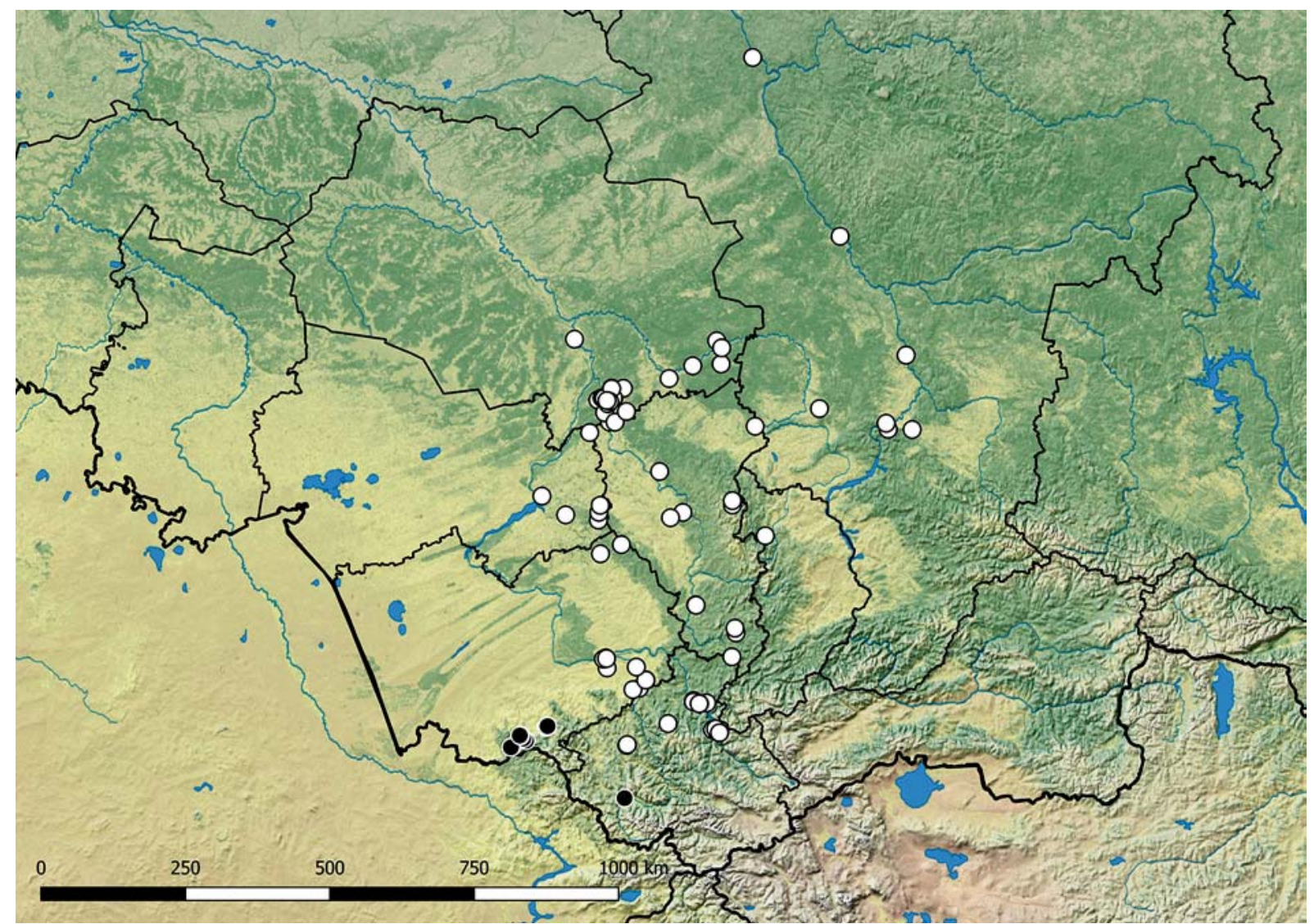

Fig. 1. Distribution of Schizoturanius species: dmitriewi in Asian Russia (black circle) and clavatipes (white circle).

Рис. 1. Распространение видов Schizoturanius: dmitriewi в азиатской части России (черный круг) и clavatipes (белый круг). 


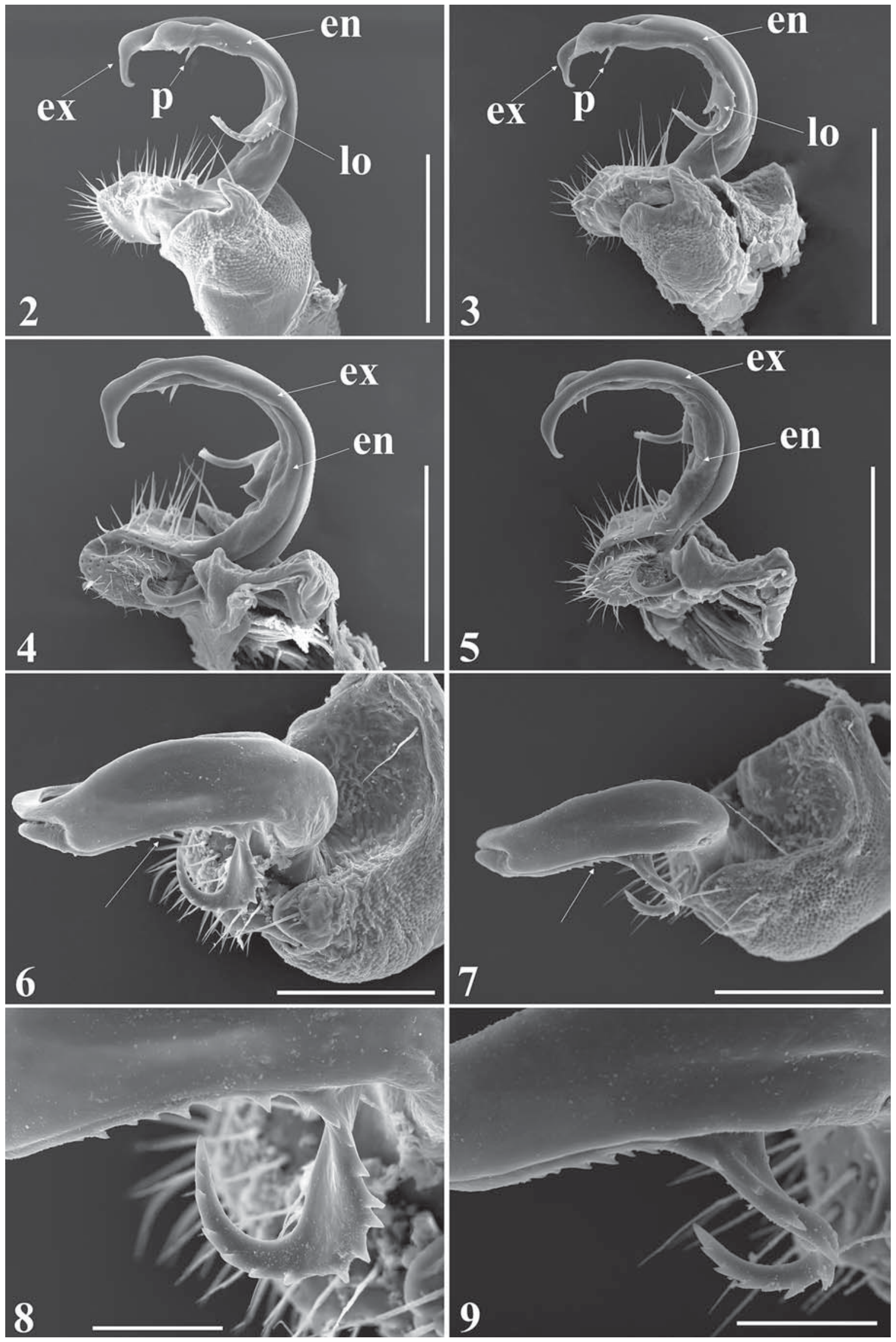




\section{Schizoturanius clavatipes (Stuxberg, 1876)}

Figs 1, 10-11.

Polydesmus clavatipes Stuxberg, 1876a: 34, inset: figs. Polydesmus clavatipes - Stuxberg, 1876b: 316; Attems, 1904: 48; Nefediev, Nefedieva, 2008a: 117.

Schizoturanius clavatipes - Lohmander, 1933: 27; Hoffman, 1975: 81, 82: figs; Lokšina, Golovatch, 1979: 384; Golovatch, 1979 : 993; Mikhaljova, 1993: 31, 32: figs; 2002: 206; 2004: 238, 239: figs, 228: map; 2013a: 221; 2013b: 9; 2016: 24; 2017: 288, 289: figs, 290 map; Mikhaljova, Golovatch, 2001: 116; Nefediev, 2001: 85; 2002c 139; Vorobiova et al., 2002: 62; Mikhaljova, Nefediev, 2003: 81 Nefediev, Nefedieva, 2005: 178; 2006: 98; 2007a: 139; 2007b: 161 2007c: 99; 2008b: 62; 2011: 100; 2012a: 47; 2012b: 51; 2013: 87; 2017: 292; Nefedieva, Nefediev, 2008: 123; Nefediev et al., 2014: 63; Nefedieva et al., 2014: 65; 2015: 152.

Turanodesmus clavatipes - Attems, 1940: 85.

Turanodesmus dmitrievi (sic!) - Byzova, Chadaeva, 1965: 337.

non Schizoturanius clavatipes - Nefediev et al., 2017: 13; 2018: 228

non Schizoturanius clavatipes pro parte - Nefediev, Nefedieva, 2017: 292.

MATERIAL EXAMINED. 2 juv. (ASU), Russia, Krasnoyarsk Province, Sorokino Railway Station, Betula forest with Pinus sibirica and Alnus, litter, 23.VIII.1994, leg. A.B. Ryvkin; $1 \sigma^{\top}$ (ASU), Russia, Tomsk Area, Tomsk District, Tomsk, "Universitetskaya Roshcha" Park, $56.468895^{\circ} \mathrm{N}, 84.949151^{\circ} \mathrm{E}$, Betula pendula forest, $105 \mathrm{~m}$ a.s.1., 19.IV.2000; $3 \sigma^{\top} \sigma^{\top}, 3$ 0 ( (ASU), same Area and District, $15 \mathrm{~km} \mathrm{~N}$ of Tomsk, mixed forest, 11.VI.2000; $1 \sigma^{\top}, 19$, 19 , 8 juv. (ASU), same Area, Teguldet District, near Novyi Trud (abandoned), $57.428549^{\circ} \mathrm{N}, 87.999207^{\circ} \mathrm{E}$, Populus tremula forest with Pinus sibirica, $175 \mathrm{~m}$ a.s.l., p.t., 15.VI-11.VII.2000; 1 ๆ', 4 오, 3 juv. (ASU), same Area and District, near Chet-Kontorka, 57. $064860^{\circ} \mathrm{N}, 88.115124^{\circ} \mathrm{E}$, Populus tremula forest with Abies sibirica and Betula pendula, $190 \mathrm{~m}$ a.s.1., 19.VI.2000; 2 juv. (ASU), near s.1., Abies sibirica forest, 19.VI.2000; 6 ㅇ, 11 juv. (ASU), same Area and District, near Teguldet, $57.316881^{\circ} \mathrm{N}, 88.139437^{\circ} \mathrm{E}$, Populus tremula forest, $135 \mathrm{~m}$ a.s.1., 4.VII.2000; 2 우, 4 juv. (ASU), same Area and District, near Chiornyi Yar, 57.04062 ${ }^{\circ} \mathrm{N}, 87$. $301126^{\circ} \mathrm{E}, 150 \mathrm{~m}$ a.s.1., 5.VII.2000, all leg. P.N.; 2 우 (ASU), same Area, Tomsk District, near Zorkaltsevo, $56.521326^{\circ} \mathrm{N}, 84.733903^{\circ} \mathrm{E}$, Pinus sibirica forest, p.t., 23.V.3.VI.2001; 1 乃, 2 90, 2 fragm. (ASU), s.1., p.t., 3-13.VI.2001; $2 \sigma^{7} \sigma^{7}, 2$ 2 (ASU), s.1., p.t., 13-26. VI.2001; 2 juv. (ASU), s.1., s.s., 16-19.VI.2001; 4 O+口, 1 fragm. (ASU), s.1., p.t., 26.VI-7.VII.2001; 14 juv., 6 fragm. (ASU), s.1. p.t., 7-24.VII.2001; 7 juv. (ASU), s.1., s.s., 24.VII.2001; 2 우, 5 fragm. (ASU), s.1., p.t., 24.VII-8.VIII.2001; 1 क, 4 fragm. (ASU), s.1., p.t., 8-24.VIII.2001; 1 O', 4 juv. (ASU), s.1., s.s., 24.VIII.2001; $6 \sigma^{\top} \sigma^{7}, 12$ 우, 1 juv., 1 fragm. (ASU), s.1., p.t., 24.VIII-20.IX.2001; $1 \sigma^{7}, 1$ (ASU), s.1., s.s., 16.X.2001; $1 \sigma^{7}, 4$ 우, 35 juv. (ASU), same Area and District, near Kislovka, $56.430654^{\circ} \mathrm{N}, 84.900971^{\circ} \mathrm{E}$, Populus tremula forest, s.s., 10-11.VI.2001; 4 कo , 3 juv. (ASU), s.1. p.t., 10-26.VI.2001; 1 9, 3 juv. (ASU), s.1., p.t., 26.VI-26.VII.2001; 47 juv., 1 fragm. (ASU), s.1., s.s., 18-22.VII.2001; 38 juv. (ASU), s.1., p.t., 26.VII-6.VIII.2001; 6 O $^{\top} \sigma^{\top}, 6$ 6 , 46 juv., 1 fragm. (ASU), s.1., p.t., 6-26.VIII.2001; $1 \sigma^{\top}, 6$ \% 6 , 34 juv., 1 fragm. (ASU), s.1., s.s., 26.VIII.2001; $10 \sigma^{7} \sigma^{7}, 19$ 90,11 juv., 1 fragm. (ASU), s.1., p.t., 26.VIII-18.IX.2001; 4 O$^{\top} \sigma^{\top}, 13$ 0 , 10 juv., 1 fragm. (ASU), s.1., s.s., 9.X.2001; 11 juv. (ASU), same Area and District, near Petrovskii Uchastok, $56.512449^{\circ} \mathrm{N}, 84.789453^{\circ} \mathrm{E}$, Populus tremula and Betula pendula patch, s.s., 20-26.VI.2001; $3 \bigcirc^{\top} \sigma^{7}, 3$ of, 14 juv., 1 fragm. (ASU), s.1., s.s., 8-10.VII.2001; 3 O $^{7}, 1$ \%, 3 juv., 1 fragm. (ASU), s.1., s.s., 24.VIII.2001; 1 † (ASU), s.1., p.t., 24.VIII.20.IX.2001; 1 ㅇ, 6 juv. (ASU), s.1., s.s., 16.X.2001; $1 \sigma^{\top}, 1$ q (ASU), same Area and District, near Timiryazevskoye, ecotone of Betula pendula forest and Pinus sylvestris forest, p.t., 26.VIII-18.IX.2001, all leg. P.N., J.N.; $1 \mathrm{O}^{7}$ (ASU), same Area, Krivosheino District, near Novoislambul, $57.418825^{\circ} \mathrm{N}, 83.878022^{\circ} \mathrm{E}$, mixed forest, $115 \mathrm{~m}$ a.s.1., 21.IX.2003, leg. P.N.; 5 juv. (ASU), same Area, Tomsk District, near Zorkaltsevo, Pinus sibirica forest, p.t., 12.VII.-24.VIII. 2006, leg. S.A. Krivets; $1 \sigma^{7}, 1$, 1 fragm. (ASU), Russia, Novosibirsk Area, Toguchin District, near Yurty, 14.VIII.2007, leg. A.S. Babenko; 1 ', 3 우, 3 juv. (ASU), Russia, Kemerovo Area, Kemerovo District, $45 \mathrm{~km}$ N of Tebenkovka, $55^{\circ} 23^{\prime} 18.11^{\prime \prime} \mathrm{N}, 86^{\circ} 22^{\prime}$ $37.29^{\prime \prime}$, Abies sibirica forest with Betula pendula and Populus tremula, $170 \mathrm{~m}$ a.s.1., 30.VIII.2016; 1 juv. (ASU), same Area, Krapivinskii District, $8 \mathrm{~km} \mathrm{SSW}$ of Saltymakovo, $54^{\circ} 45^{\prime} \mathrm{N}, 87^{\circ} 01^{\prime} \mathrm{E}$, Kemerovo State University Field Station "Azhendarovo", floodplain of Tom River, $165 \mathrm{~m}$ a.s.1., 28.VII.2017; $1 \sigma^{\Upsilon}, 5$ 우 (ASU), same Area and District, $56 \mathrm{~km} \mathrm{~S}$ of Taradanovo, $54^{\circ} 40 \mathrm{~N}, 86^{\circ} 41^{\prime} \mathrm{E}$, Populus tremula forest, $300 \mathrm{~m}$ a.s.l., in litter and rotten logs, 13 . VIII.2017, all leg. D.A. Efimov; 2 juv. (ASU), Russia, Altai Province, Zalesovo District, at border with Kemerovo Area, $54.235533^{\circ} \mathrm{N}$, $85.375949^{\circ} \mathrm{E}$, Betula pendula forest with Ribes nigrum, $400 \mathrm{~m}$ a.s.1., 3.VII.2018, leg. P.N.; 1 juv. (ASU), Russia, Republic of Altai, Shebalino District, 3.5 air-km SE of Topuchaya, $51.11189^{\circ} \mathrm{N}$, $85.63219^{\circ} \mathrm{E}$, Betula pendula, Larix sibirica, Pinus sibirica and Picea obovata forest with Alnus, Lonicera, Ribes nigrum and tall grass vegetation on hummocks, along brook, ca. $1435 \mathrm{~m}$ a.s.1., sifted leaf litter, 22.VII.2018, leg. V.G., M.M., V.L.

DISTRIBUTION. This species is widespread in southwestern Siberia (SE districts of the Tomsk Area, E districts of the Novosibirsk Area and Altai Province, $\mathrm{N}$ and central districts of the Republic of Altai, the entire Kemerovo Area, NW districts of the Republic of Khakassia) and the southwest of central Siberia (SW districts of the Krasnoyarsk Province) (Fig. 1).

REMARKS. Originally described in Polydesmus Latreille, 1802-1803 from the territory between Achinsk (Krasnoyarsk Province) and Mariinsk (Kemerovo Area) [see Stuxberg, 1876a, b], this species was later transferred to Schizoturanius [Lohmander, 1933].

Being very close to $S$. dmitriewi, $S$. clavatipes differs from it and other species of the genus mainly by the presence of a small triangular blade (b) at the inner edge of the middle part of the gonopod exomere (ex) (Fig. 11), as well as the smooth, non-dentate outer edge of the gonopod endomere (en), which is directed caudad, elongated and distally pointed, carrying a small, thin process (p) on the inner side near the pad/pulvillus (Fig. 10).

These two species are also quite similar in habitus, but due to their non-overlapping distribution areas even their females and juveniles are easily distinguishable. Living together over much of their distribution ranges, S. clavatipes and $S$. tabescens are not only sympatric, but often even syntopic congeners (Figs 1, 12).

\section{Schizoturanius tabescens (Stuxberg, 1876)}

Fig. 12 .

Polydesmus tabescens Stuxberg, 1876a: 35, inset: figs. Polydesmus tabescens - Stuxberg, 1876b: 316; Attems, 1904: 48. Turanodesmus salairicus Gulička, 1963: 523, 522: figs. Turanodesmus salairicus - Byzova, Chadaeva, 1965: 333; Nefediev, Nefedieva, 2008a: 117; Babenko et al., 2009: 182.

Schizoturanius salairicus — Lokšina, Golovatch, 1979: 384; Mikhaljova, 1993: 31; Mikhaljova, Golovatch, 2001: 116; Nefedi-

Figs 2-9. Schizoturanius dmitriewi, males: 2-7 - gonopod; 8-9 - lateral outgrowth of gonopod endomere; 2-3 - lateral view; 4-5 mesal view; 6-9 - front and ventral view; 2, 4, 6, 8- Voronezh Nature Reserve; 3, 5, 7, 9 - Altai Province, near Charyshskoye. Scale bar: $0.4 \mathrm{~mm}(2-5), 0.3 \mathrm{~mm}(6-7), 0.1 \mathrm{~mm}(8-9)$. Designations explained in text.

Рис. 2-9. Schizoturanius dmitriewi, самцы: 2-7 - гонопод; 8-9 - боковой отросток эндомера гонопода; 2-3 - вид сбоку; 4-5 вид изнутри; 6-9 - вид спереди и снизу; 2, 4, 6, 8- Воронежский заповедник; 3, 5, 7, 9 - Алтайский край, около Чарышского. Масштаб: 0,4 мм (2-5), 0,3 мм (6-7), 0,1 мм (8-9). Объяснение обозначений дано в тексте. 


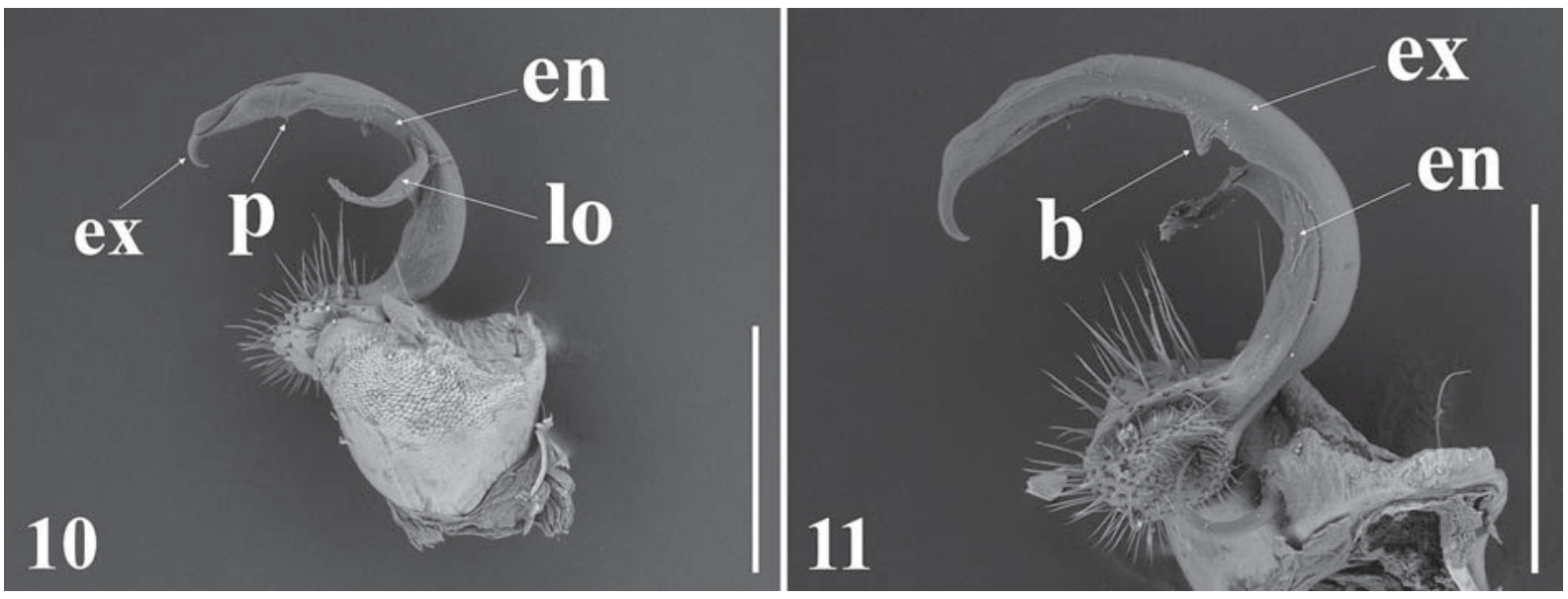

Figs 10-11. Schizoturanius clavatipes, male (Altai Province, near Zalesovo): 10 - gonopod, lateral view; 11 — gonopod, mesal view. Scale bar: $0.5 \mathrm{~mm}$. Designations explained in text.

Рис. 10-11. Schizoturanius clavatipes, самец (Алтайский край, около Залесово): 10 — гонопод, сбоку; 11 — гонопод, изнутри. Масштаб: 0,5 мм. Объяснение обозначений дано в тексте.

ev, 2001: 85; 2002a: 41; 2002b: 30; 2002c: 139; 2002d: 35; Mikhaljova, Nefediev, 2003: 83; Nefediev, Nefedieva, 2008a: 118 .

Schizoturanius tabescens Mikhaljova, 1993: 31, 32: figs; 2004:

240, 241: figs, 242: map; 2013a: 221; 2017: 291, figs, 292: map; Vorobiova, 1999: 33; Mikhaljova, Golovatch, 2001: 116; Vorobiova et al., 2002: 62; Rybalov, 2002: 83; Rybalov, Vorobiova, 2002: 14 Mikhaljova, Marusik, 2004: 8, 7: figs; Nefediev, Nefedieva, 2005: 178; 2006: 98; 2007a: 139; 2007b: 161; 2007c: 100; 2008a: 118; 2008b: 62; 2011: 100; 2012a: 51; 2012b: 47; 2013: 87; 2017: 292; Nefedieva, Nefediev, 2008: 123; Babenko et al., 2009: 183; Bukhkalo et al., 2014: 74; Nefedieva et al., 2014: 65; 2015: 153.

MATERIAL EXAMINED. 1 \& (ASU), Russia, Krasnoyarsk Province, Sorokino Railway Station, Betula forest with Pinus sibir$i c a$ and Alnus, in litter, 23.VIII.1994, leg. A.B. Ryvkin; 1 ㅇ (ASU), Russia, Tomsk Area, Tomsk District, $15 \mathrm{~km} \mathrm{~N}$ of Tomsk, mixed forest, 11.VI.2000; 9 우, 4 juv., 2 fragm. (ASU), same Area,

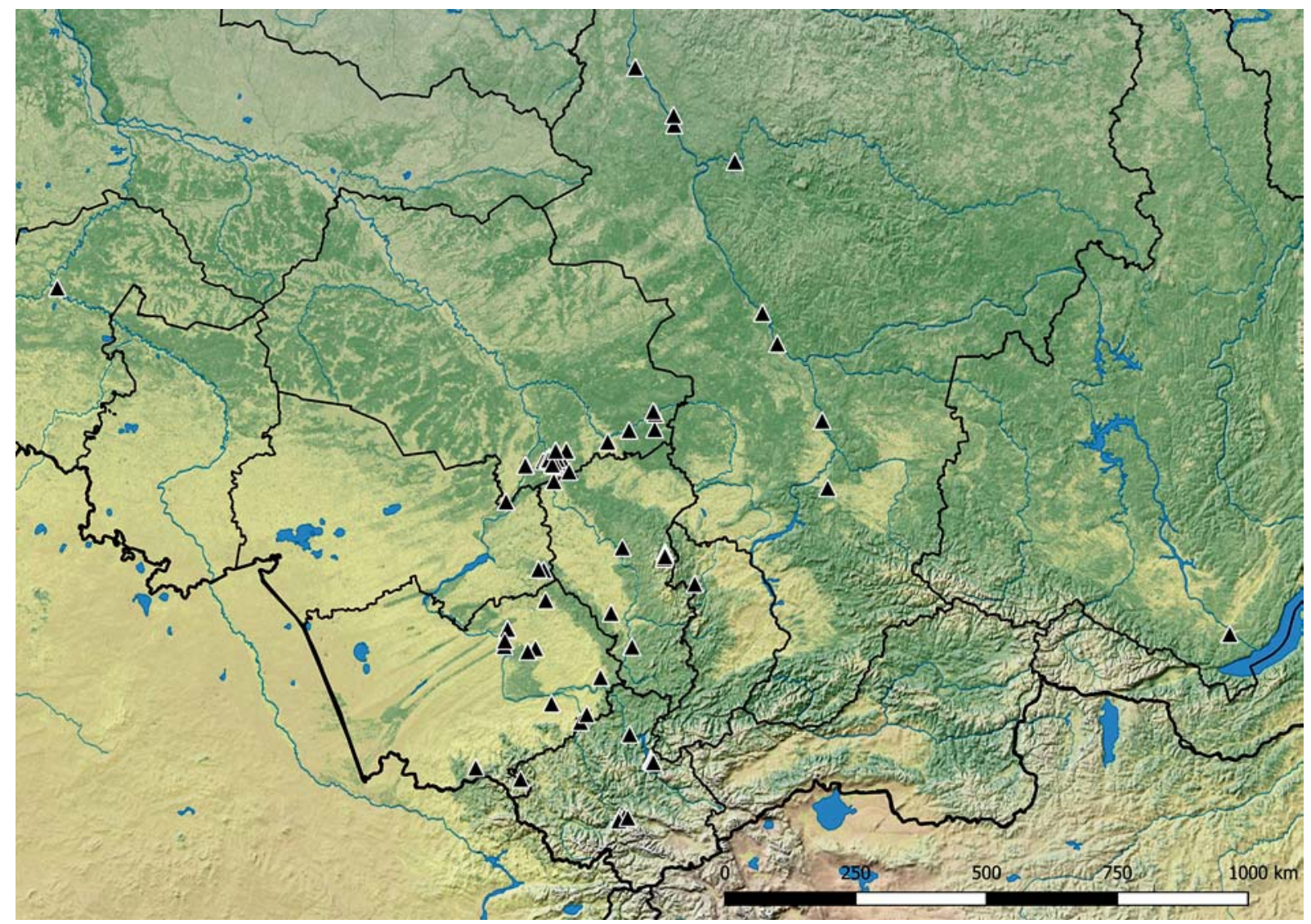

Fig. 12. Distribution of Schizoturanius tabescens.

Рис. 12. Распространение Schizoturanius tabescens. 
Teguldet District, near Chet-Kontorka, $57.064860^{\circ} \mathrm{N}, 88.115124^{\circ} \mathrm{E}$ Populus tremula forest, $190 \mathrm{~m}$ a.s.1., 19.VI.2000; 4 juv. (ASU), same Area and District, near Sosnovka (abandoned), $57.363366^{\circ} \mathrm{N}$ $88.073710^{\circ} \mathrm{E}$, Betula pendula and Abies sibirica forest, $135 \mathrm{~m}$ a.s.1., h.s., 22.VI.2000; 19 OO, 9 juv. (ASU), same Area and District, near Teguldet, $57.316881^{\circ} \mathrm{N}, 88.139437^{\circ} \mathrm{E}$, Populus tremula forest, 135 m a.s.1., 4.VII.2000; 1 juv. (ASU), same Area and District, nea Chiornyi Yar, $57.040621^{\circ} \mathrm{N}, 87.301126^{\circ} \mathrm{E}, 150 \mathrm{~m}$ a.s.1., 5.VII.2000, all leg. P.N.; 4 ㅇ, 4 juv. (ASU), same Area, Tomsk District, near Zorkaltsevo, $56.521326^{\circ} \mathrm{N}, 84.733903^{\circ} \mathrm{E}$, Pinus sibirica forest, s.s. 16-19.VI.2001; 5 +9, 34 juv., 1 fragm. (ASU), s.1., s.s., 24.VII.2001; 14 juv. (ASU), s.1., s.s., 24.VIII.2001; 59 우, 3 juv., 2 fragm. (ASU), s.1., s.s., 16.X.2001; 1 क, 2 juv. (ASU), same Area and District, near Kislovka, $56.430654^{\circ} \mathrm{N}, 84.900971^{\circ} \mathrm{E}$, Populus tremula forest, s.s. 10-11.VI.2001; 22 juv., s.1., s.s., 18-22.VII.2001; 1 +, 14 juv. (ASU), s.1., s.s., 26.VIII.2001; 19 +9, 13 juv., 1 fragm. (ASU), s.1., s.s., 9.X.2001; 35 oᄋ, 356 juv., 5 fragm. (ASU), same Area and District, near Petrovskii Uchastok, 56.512449 ${ }^{\circ} \mathrm{N}, 84.789453^{\circ} \mathrm{E}$, Populus tremula and Betula pendula patch, s.s., 20-26.VI.2001; 13 juv. (ASU), s.1., s.s., 8-10.VII.2001; 12 कq, 13 juv., 1 fragm. (ASU), s.1., s.s., 24.VIII.2001; 7 + (ASU), s.1., p.t., 24.VIII-20.IX.2001; 29 90,3 juv. (ASU), s.1., s.s., 16.X.2001, all leg. P.N., J.N.; 7 (ASU), same Area and District, Tomsk, "Buff-Sad" Park, $56.471503^{\circ} \mathrm{N}, 84.962563^{\circ} \mathrm{E}$, Acer negundo and Populus, $110 \mathrm{~m}$ a.s.1., 15.V.2008, leg. P.N.; 1 juv. (ASU), same Area and District, near Zorkaltsevo, Pinus sibirica forest, p.t., 12.VII-24.VIII.2006, leg. S.A. Krivets; 1 , 1 fragm. (ASU), Russia, Kemerovo Area Krapivinskii District, near Zelenogorskii, $55^{\circ} 01 \mathrm{~N}, 87^{\circ} 05^{\prime} \mathrm{E}$, bank of Tom River, under bark, 140 m a.s.1., h.s., 9.VII.2017, leg. D.A. Efimov; 1 (ASU), Russia, Altai Province, Barnaul, "Lesnaya Skazka" Park, $53.359283^{\circ} \mathrm{N}, 83.682242^{\circ} \mathrm{E}$, Acer negundo and Betula pendula stand, under boards, pieces of concrete, in litter, $215 \mathrm{~m}$ a.s.1., 1.V.2015, leg. P.N.; 1 क (ASU), same Province, Charyshskoye District, ca. $4.5 \mathrm{~km}$ SE of Charyshskoye, environs of ASU Field Station "Goluboi Utios", 51²1'33.8'N, 8337'23.2'E, Betula pendula and Populus tremula stand on N slope, ca. $520 \mathrm{~m}$ a.s.1., 5.VI. 2018, leg. Kh.N., S.N., V.S.; 1 (ASU), near s.1., $51.362106^{\circ}$ N, 83 $623658^{\circ} \mathrm{E}$, Salix thicket in valley of Pikhtovka River, $460 \mathrm{~m}$ a.s.1., 8.VI.2018, leg. Kh.N.; 1 (ASU), same Province and District, $5 \mathrm{~km}$ $\mathrm{S}$ of Ust-Kumir, $50^{\circ} 58.988^{\prime} \mathrm{N}, 84^{\circ} 17.415^{\prime} \mathrm{E}$, valley of Kumir River, forest with gaps of tall herbaceous vegetation, ca. $805 \mathrm{~m}$ a.s.1., sifting leaf litter, 1.VIII.2018, leg. V.G., M.M., V.L.; 1 juv. (ASU), Russia, Republic of Altai, Ongudai District, 9 air-km SSW of Belyi Bom, near Achik Pass, 50.295678 $\mathrm{N}, 86.977943^{\circ} \mathrm{E}$, sparse Pinus sibirica forest edge, $2120 \mathrm{~m}$ a.s.1., 21.VII.2006, leg. P.N., J.N.; 1 juv. (ASU), same Republic and District, 20 air-km W of Chibit, near Shirlak Waterfall, valley of Chuya River, right bank of Chuya River, Betula forest with Larix sibirica, Caragana arborescens, low grasses and green mosses, $50.34358^{\circ} \mathrm{N}, 87.22225^{\circ} \mathrm{E}$, ca. $1015 \mathrm{~m}$ a.s.1., 23.07.2018, leg. P.N.; 1 9 (ASU), Russia, Irkutsk Area, Irkutsk, Akademgorodok, left bank of Angara River, 52 $144^{\prime} 48.1^{\prime \prime} \mathrm{N}, 104^{\circ}$ $15^{\prime} 13.4^{\prime \prime}$, park forest with Betula and Populus tremula, seldom Pinus sylvestris, Alnus fruticosa and Swida alba, ca. $520 \mathrm{~m}$ a.s.1., h.s., 31.VIII.2018, leg. I.V. Enushchenko.

DISTRIBUTION. This species is widespread in southwestern and central Siberia (SE districts of the Tomsk Area, E districts of the Novosibirsk Area, N, E and S districts of the Altai Province, central and S districts of the Kemerovo Area, $\mathrm{N}$ and central districts of the Republic of Altai, NW districts of the Republic of Khakassia, and SW districts of the Krasnoyarsk Province). The species is also known a significant distance away from the main part of its distribution area, being recorded from the south of the Tyumen and Irkutsk areas as well (Fig. 12).

REMARKS. Originally described in Polydesmus from the Yenisei River valley near Yeniseisk and Alinskoye (both Krasnoyarsk Province) [Stuxberg, 1876a, b], this species was later transferred to Schizoturanius [Mikhaljova, 1993] and synonymized with Turanodesmus salairicus Gulička, 1963 [Mikhaljova, Marusik, 2004]. Schizoturanius tabescens is new to be reported from the Irkutsk Area, its easternmost range limit.

Sometimes occurring even syntopically with $S$. dmitriewi at the southwestern periphery of its distribution area, $S$. tabescens can easily be distinguished from it by the smaller body and lighter coloration (see above), same as from $S$. clavatipes, with which $S$. tabescens is a sympatric species.

The following key can be proposed to Schizoturanius species from Asian Russia.

1(2) Gonopod broadened, with a short exomere. Gonopod endomere without a lateral outgrowth ......... tabescens

2(3) Gonopod elongated and crescent, with an exomere longer than endomere. Gonopod endomere with a lateral outgrowth (lo) (Figs 3, 10)

3(4) Outer edge of gonopod endomere smooth, non-dentate clavatipes

4 (3) Outer edge of gonopod endomere serrate (Figs 8-9) .. dmitriewi

\section{Conclusions}

At least three species of Schizoturanius are currently known to occur in Asian Russia. All presently known records of $S$. dmitriewi from the Asian part of Russia are provided, where the species is found in the Republic of Altai for the first time. The easternmost, new records of S. tabescens from the Irkutsk Area are also given.

A direct comparative re-examination of the gonopods of $S$. dmitriewi from a European population with specimens from the southwestern Siberian populations shows their identity (Figs 2-9). Hence, S. dmitriewi reveals a disjunct distribution area, being among the very few examples of millipede migration from Siberia to the Russian Plain. These two species are close, but differ from each other and their congeners mainly by the presence or absence of a small triangular blade (b) at the inner edge of the middle part of the exomere (ex) (Fig. 11 vs. Figs 4-5), and by a smooth or serrate outer edge of the endomere (en) (Fig. $10 v s$. Figs 8-9); also the tip of the endomere can be elongated and distally pointed or shortened and swollen ventrally (Fig. 10 vs. Fig. 2), but anyway it carries a thin, more or less elongated process (p) near the pad/pulvillus (Figs 2-3, 10).

All three Schizoturanius species inhabiting the Asian part of Russia can easily be distinguished from each other taking into account (1) the habitus, i.e. body size and coloration, and (2) distribution areas. Thus, $S$. tabescens, being sympatric with $S$. clavatipes and occasionally dwelling syntopically with $S$. dmitriewi, differs easily from both by the smaller body and white coloration. At the same time, $S$. dmitriewi and $S$. clavatipes, being very similar in habitus and having non-overlapping distribution areas, are fully allopatric.

Acknowledgements. I am very thankful to S.I. Golovatch (Moscow, Russia) who kindly edited the text and checked its English. I am most grateful to Arkady A. Schileyko and E.N. Kudryavtseva (both ZMUM) for the access to the ZMUM diplopod collection under their care. My deepest gratitude is extended to all persons who provided material for 
the present study: T.M. Krugova and Yu.V. Dyachkov (both Barnaul, Russia), D.A. Efimov (Kemerovo, Russia), V.I. Gusarov (Oslo, Norway), A.S. Babenko and S.A. Krivets (both Tomsk, Russia), and G.Sh. Farzalieva (Perm, Russia). I am highly obliged to R.Yu. Dudko (ISEA) and V.V. Kirillov (IWEP) who kindly provided the facilities for taking SEM micrographs.

\section{References}

Attems C. 1904. Myriopoden // Fauna Arctica. Bd.3. S.33-54. Attems C. 1940. Myriapoda 3. Polydesmoidea III. Fam. Polydesmidae, Vanhoeffeniidae, Cryptodesmidae, Oniscodesmidae, Sphaerotrichopidae, Peridontodesmidae, Rhachidesmidae, Macellolophidae, Pandirodesmidae // Das Tierreich. Lfg.70. S.1-577.

Babenko A.S., Nefediev P.S., Nefedieva J.S. 2009. [The fauna and population dynamics of the millipedes (Diplopoda) of the Salair chern taiga] // Vestnik Tomskogo gosudarstvennogo universiteta, Seriya Biologiya. No.319. P.182-185 [in Russian].

Bukhkalo S.P., Galitch D.E., Sergeeva E.V., Vazhenina N.V. 2014 [A synopsis of the invertebrate fauna in the southern taiga of Western Siberia (basin of the Lower Irtysh River)]. Moscow: KMK Scientific Press. 189 pp. [In Russian]

Byzova Yu.B., Chadaeva Z.V. 1965. [Comparative characteristics of soil fauna of various associations in an Abies sibirica forest (Kemerovo Area)] // Zoologicheskii Zhurnal. Vol.44. No.3. P.331-339 [in Russian, with English summary].

Evsyukov A.P., Golovatch S.I. 2013. Millipedes (Diplopoda) from the Rostov-on-Don Region, southern Russia // Arthropoda Selecta. Vol.22. No.3. P.207-215.

Golovatch S.I. 1979. [The composition and zoogeographic relationships of the diplopod fauna of Middle Asia. Part 1] // Zoologicheskii Zhurnal. Vol.58. No.7. P.987-1001 [in Russian, with English summary].

Golovatch S.I. 1984. [Distribution and faunogenesis of millipedes in the USSR European part] // Y.I. Chernov (Ed.), Faunogenez filotsenogenez. Moscow: Nauka. P.92-138 [in Russian].

Golovatch S.I. 1992. Some patterns in the distribution and origin of the millipede fauna of the Russian Plain (Diplopoda) // Berichte des naturwissenschaftlich-medizinischen Vereins Innsbruck. Suppl.10. P.363-373.

Golovatch S.I. 2014. Review of the millipede genus Epanerchodus Attems, 1901 in continental China, with descriptions of new species (Diplopoda: Polydesmidae) // Zootaxa. Vol.3760. No.2. P. 275-288.

Golovatch S.I. 2015. Two new species of the millipede order Polydesmida from Southern China (Diplopoda) // Zoologicheskii Zhurnal. Vol.94. No.9. P.1023-1028.

Gulička J. 1963. [New millipedes (Diplopoda) from the USSR. Part 1] // Zoologicheskii Zhurnal. Vol.42. No.4. P.518-524 [in Russian, with English summary].

Hoffman R.L. 1975. A note on the status of Polydesmus clavatipes and some related species from Central Asia (Diplopoda, Polydesmidae) // Zool. Anz. Bd.194. H.1/2. S.79-83.

Krugova T.M., Nefediev P.S. 2018. [Distribution of the millipedes (Diplopoda) along an altitudinal transect in the low mountains of the northwestern Altais (Tigirek Strict Reserve), with notes on their ecological preferences and distribution area] // Trudy Tigirekskogo zapovednika. Vol.10. P.108-122 [in Russian, with English summary].

Lohmander H. 1933. Über Diplopoden aus Zentralasien // Ark. Zool. Bd.25A. Nr.6. S.1-71.

Lokshina I.E. 1966. [An analysis of the distribution of millipedes (Diplopoda) over the Russian Plain in a zonal aspect] // Zoologicheskii Zhurnal. Vol.45. No.12. P.1773-1778 [in Russian, with English summary]

Lokšina I.E., Golovatch S.I. 1979. Diplopoda of the USSR fauna // Pedobiologia. Bd.19. S.381-389.

Mikhaljova E.V. 1993. The millipedes (Diplopoda) of Siberia and the Far East of Russia // Arthropoda Selecta. Vol.2. No.2. P.3-36.
Mikhaljova E.V. 2002 [for 2001]. On some poorly-known millipedes from Siberia (Diplopoda) // Arthropoda Selecta. Vol.10. No.3. P.201-207.

Mikhaljova E.V. 2004. The millipedes (Diplopoda) of the Asian part of Russia. Sofia-Moscow: Pensoft Publishers. Series Faunistica 39. 292 pp.

Mikhaljova E.V. 2013a. [Endemism of the millipedes (Diplopoda) of the Asian part of Russia] // Izuchenie, sokhranenie i vosstanovlenie estestvennykh landshaftov. Materialy III Mezhdunarodnoi nauchno-prakticheskoi konferentsii. Volgograd. Moscow: Planeta Publ. P.220-223 [in Russian].

Mikhaljova E.V. 2013b. New data on the millipede fauna (Diplopoda) of Altai, Russia // Far Eastern Entomologist. No.265. P.1-10.

Mikhaljova E.V. 2016. New species and new records of millipedes (Diplopoda) from the Asian part of Russia // Far Eastern Entomologist. No.316. P.1-25.

Mikhaljova E.V. 2017. [The millipede fauna (Diplopoda) of the Asian part of Russia]. Vladivostok: Dalnauka Publ. 336 pp. [In Russian, with English summary]

Mikhaljova E.V., Golovatch S.I. 2001 [for 2000]. A review of the millipede fauna of Siberia (Diplopoda) // Arthropoda Selecta. Vol.9. No.2. P.103-118.

Mikhaljova E.V., Marusik Y.M. 2004. New data on taxonomy and fauna of the millipedes (Diplopoda) from the Russian Far East, Siberia and Mongolia // Far Eastern Entomologist. No.133. P.1-12.

Mikhaljova E.V., Nefediev P.S. 2003 [for 2002]. A contribution to the millipede fauna of Siberia (Diplopoda) // Arthropoda Selecta. Vol.11. No.1. P.81-87.

Nefediev P.S. 2001. [On the fauna and ecology of Myriapoda in the environs of the village of Smolenskoe, Altai Province] // Landshafty Zapadnoy Sibiri: problemy issledovaniy, ekologiya i ratsionalnoe ispolzovanie. Materialy VII Mezhdunarodnoy mezhvuzovskoy konferentsii, posvyaschjonnoy Dnyu Zemli. Biysk: Biysk Pedagogical State University Publ. P.84-86 [in Russian].

Nefediev P.S. 2002a. [Eco-faunistic investigations of myriapods in the Teguldet District, Tomsk Area] // Materialy Mezhdunarodnoi konfrentsii studentov i molodykh uchionykh "Lomonosov2002”. Vol.7. Moscow: Moscow State University Publ. P.40-41 [in Russian].

Nefediev P.S. 2002b. On the Diplopoda fauna of the South-West Siberia // Abstracts of 12th International Congress of Myriapodology. 28 July-3 August 2002, Mtunzini, KwaZulu-Natal, South Africa. P.30.

Nefediev P.S. 2002c. [Population and some ecological peculiarities of myriapods of gray forest soils in the south of the Tomsk Area] // Biology, a science of the XXI century. Materialy 6 shkolykonferentsii molodykh uchionykh. Pushchino-on-Oka: Pushchino Scientific Center RAS Publ., P.138-139 [in Russian].

Nefediev P.S. 2002d. [The fauna and ecology of myriapods (Myriapoda) of a relict lime grove (village of Kuzedeyevo)] // Student i nauchno-tekhnicheskii progress: Biologiya. Materialy XL Mezhdunarodnoi nauchnoi studencheskoi konferentsii. Novosibirsk: Novosibirsk State University Publ. P.35 [in Russian].

Nefediev P.S., Dyachkov Yu.V., Nefedieva J.S. 2014. Fauna and ecology of millipedes (Diplopoda) in the Tigirek State Nature Reserve, Russian Altai // I.H. Tuf, K. Tajovský (eds.). 16th International Congress of Myriapodology. Book of Abstracts. Olomouc: Institute of Soil Biology, BC ASCR \& Faculty of Science, Palacký University. P.63.

Nefediev P.S., Farzalieva G.Sh., Tuf I.H., Nedoev H.Kh., Niyazov S.T. 2017. Millipede and centipede assemblages on the northern and southern slopes of the lowland Altais, southwestern Siberia, Russia (Diplopoda, Chilopoda) // Tropical Natural History. Suppl.5. Book of abstracts. 17th International Congress of Myriapodology. 23-26 July 2017, Krabi, Thailand. P.13.

Nefediev P.S., Farzalieva G.Sh., Tuf I.H., Nedoev H.Kh., Niyazov S.T. 2018. Millipede and centipede assemblages on the northern and southern slopes of the lowland Altais, southwestern Siberia, Russia (Diplopoda, Chilopoda) // P. Stoev, G.D. Edgecombe (eds). Proceedings of the 17th International Congress of Myriapodology, Krabi, Thailand. ZooKeys. No.741. P.219-254. doi: 10.3897/zookeys.741.21936. 
Nefediev P.S., Nefedieva J.S. 2005. [Seasonal fluctuations of diplopod density in some forests of Western Siberia] // B.R. Striganova (ed.). Ekologicheskoye raznoobrazie pochvennoi bioty i biologicheskaya produktivnost pochv. Materialy dokladov IV (XIV) Vserossiiskoi konferentsii po pochvennoi zoologii. 1-4 February 2005, Tyumen. Tyumen: Tyumen State University Publ. P.177-178 [in Russian]

Nefediev P.S., Nefedieva J.S. 2006. [Regional peculiarities of the millipede fauna (Diplopoda) in the south-east of Western Siberia] // V.V. Anyushkin (ed.). Ekologiya Yuzhnoi Sibiri i sopredelnykh territorii. Sbornik materialov X Mezhdenarodnoi shkoly-konferentsii studentov i molodykh uchionykh. Abakan: Khakassian State University Publ. Vol.10. No.1. P.98 [in Russian].

Nefediev P.S., Nefedieva J.S. 2007a. A brief analysis of the biotopic distribution of millipedes (Diplopoda) in the south-east of Western Siberia // A.I. Taskaev (ed.). Lesnoye pochvovedenie: itogi, problem, perspektivy. Tezisy dokladov Mezhdunarodnoi nauchnoi konferentsii. 4-11 September 2007, Syktyvkar. Syktyvkar: Institute of Biology, Komi Scientific Center, Ural Division of RAS Publ. P.139-140.

Nefediev P.S., Nefedieva J.S. 2007b. [Biogeographical characteristic of the millipede fauna in the southeast of Western Siberia] // G.P. Ostroverkhova (ed.). Bioraznoobrazie bespozvonochnykh zhivotnykh. Sbornik materialov II Vserossiiskoi shkoly-seminara s mezhdunarodnym uchastiem. 24-26 October 2007, Tomsk. Tomsk: Deltaplan Publ. P.159-164 [in Russian].

Nefediev P.S., Nefedieva J.S. 2007c. [Seasonal dynamics of locomotor activity of the millipedes (Diplopoda) in forests of Western Siberia] // V.N. Kazin (ed.). Ekologicheskie problemy unikalnykh prirodnykh i antropogennykh landshaftov. Sbornik materialov Vserossiiskoi nauchno-prakticheskoi konferentsii. 29 November 2007, Yaroslavl. Yaroslavl: Yaroslavl State University Publ. P.98-103 [in Russian].

Nefediev P.S., Nefedieva J.S. 2008a. [A historical review of faunistic investigations of the millipedes (Diplopoda) in western Siberia] // V.M. Vazhov (ed.). Altai: ekologiya i prirodopolzovanie. Trudy VII rossiisko-mongolskoi nauchnoi konferentsii molodykh uchionykh i studentov. Vol.1. Biysk: Biysk Pedagogical State University Publ. P.117-120 [in Russian].

Nefediev P.S., Nefedieva J.S. 2008b. Zoogeographical analysis of the millipede fauna (Diplopoda) in the south-east of Western Siberia // Myriapoda and Onychophora of the World Diversity, Biology and Importance. Abstracts of 14th International Congress of Myriapodology. Staatliches Museum für Naturkunde, Görlitz. Peckiana. Vol.6. P.62.

Nefediev P.S., Nefedieva J.S. 2011. [Millipedes (Diplopoda) of green plantations of the city of Tomsk and its suburbs] // Kontseptualnye i prikladnye aspekty nauchnykh issledovanii v oblasti zoologii bespozvonochnykh. Sbornik materialov III Vserossiiskoi shkoly-seminara s mezhdunarodnym uchastiem, posvyaschionnoi 120-letiyu so dnya rozhdeniya Rostislava Petrovicha Berezhkova (1891-1961). 24-27 October 2011, Tomsk. Tomsk: Agraf-Press Publ. P.100-102 [in Russian]

Nefediev P.S., Nefedieva J.S. 2012a. [Some peculiarities of the distribution of the millipedes (Diplopoda) along a soil profile in the south-taiga and small-leaved forests of Western Siberia // Izvestiya Altaiskogo gosudarstvennogo universiteta, Biologicheskie nauki. Vol.3(75). No.1. P.49-54 [in Russian, with English summary].

Nefediev P.S., Nefedieva J.S. 2012b. [Some peculiarities of the seasonal dynamics of sex-age structure in millipede populations (Diplopoda) in the southern taiga and small-leaved forests of Western Siberia // Izvestiya Altaiskogo gosudarstvennogo universiteta, Biologicheskie nauki. Vol.3(75). No.2. P.46-48 [in Russian, with English summary].

Nefediev P.S., Nefedieva J.S. 2013. [Biodiversity and ecology of the millipedes in the environs of Lake Teletskoye (Diplopoda)] // Izvestiya Altaiskogo gosudarstvennogo universiteta, Biolog- icheskie nauki. Vol.3(79). No.1. P.86-87 [in Russian, with English summary].

Nefediev P.S., Nefedieva J.S. 2017. New data on the millipede fauna of the Russian Altais, southwestern Siberia (Diplopoda)// Arthropoda Selecta. Vol.26. No.4. P.288-296.

Nefediev P.S., Nefedieva J.S. 2018. [Taxonomic richness of Diplopoda of the Russian Altai] // Materialy dokladov XVIII Vserossiiskogo soveshchaniya po pochvennoi zoologii. 22-26 October 2018, Moscow, IEE RAS. Moscow: KMK Scientific Press. P.141-142 [in Russian].

Nefedieva J.S., Nefediev P.S. 2008. Ecofaunistical investigations of millipedes (Diplopoda) in the environs of Lake Teletskoe // Myriapoda and Onychophora of the World Diversity, Biology and Importance. Abstracts of 14th International Congress of Myriapodology. Staatliches Museum für Naturkunde, Görlitz. Peckiana. Vol.6. P.123-124.

Nefedieva J.S., Nefediev P.S., Sakhnevich M.B., Dyachkov Yu.V. 2014. Distribution of millipedes (Diplopoda) along an altitudinal gradient in the south of Lake Teletskoye, Altai Mts // I.H. Tuf, K. Tajovský (eds.). 16th International Congress of Myriapodology. Book of abstracts. Institute of Soil Biology, BC ASCR \& Faculty of Science, Palacký University, Olomouc. P. 65 .

Nefedieva J.S., Nefediev P.S., Sakhnevich M.B., Dyachkov Yu.V. 2015. Distribution of millipedes (Diplopoda) along an altitudinal gradient in the south of Lake Teletskoye, Altai Mts, Russia // ZooKeys. Vol.510. P.141-161.

Rybalov L.B. 2002. [Zonal-landscape change of soil invertebrate population in the Yenisei Region of Middle Siberia and a role of temperature adaptation in meridional (zonal) distribution of invertebrates] // Russian Entomological Journal. Vol.11. No.1. P.77-86 [in Russian, with English summary].

Rybalov L.B., Vorobiova I.G. 2002. [Population of soil invertebrates in taiga ecosystems of the midflow of the Yenisei River // Izuchenie, sokhranenie i vosstanovlenie bioraznoobraziya ekosistem na Yeniseiskom ekologicheskom transekte: Zhivotnyi mir, etno-ekologicheskie issledovniya. Vol.2. Moscow: IEE RAS Publ. P.8-42 [in Russian].

Stuxberg A. 1876a. Myriopoder från Sibirien och Waigatsch ön samlade under Nordenskiöldska expeditionen 1875 // Öfversigt af Kongliga Vetenskaps-Akademiens Förhandlingar. Årg.33. No.2. S.11-38.

Stuxberg A. 1876b. On the Myriopoda, from Siberia and Waigatsch Island, collected during the expedition of Prof. Nordenskiöld, 1875 // Annals and Magazine of Natural History. Vol.4. No.17. P.306-318. doi: 10.1080/00222937608681955.

Timotheew T.E. 1897. [Two new species of Diplopoda] // Trudy Obshchestva ispytatelei prirody pri Kharkovskom universitete. Vol.31. P.319 [in Russian].

Vorobiova I.G. 1999. [An ecofaunistic characteristic of the myriapod population (Myriapoda) in the midflow region of the Yenisei River] // B.R. Striganova (ed.). Problemy pochvennoi zoologii. Bioraznoobrazie i zhizn pochvennoi sistemy. Materialy II(XII) Vserossiiskogo soveshchaniya po pochvennoi zoologii. Moscow: KMK Scientific Press. P.33 [in Russian].

Vorobiova I.G., Rybalov L.B., Rossolimo T.E., Zalesskaja N.T. 2002. [Zonal and landscape distribution of the myriapod fauna and populations (Myriapoda) in the Yenisei River basin] // Izuchenie, sokhranenie i vosstanovlenie bioraznoobraziya ekosistem na Yeniseiskom ekologicheskom transekte: Zhivotnyi mir, etno-ekologicheskie issledovniya. Vol.2. Moscow: IPEE RAS Publ. P.60-71 [in Russian].

Wytwer J., Golovatch S.I., Penev L. 2009. Variation in millipede (Diplopoda) assemblages in oak woodlands of the Eastern European Plain // Soil Organisms. Vol.81. No.3. P.791-813.

Zalesskaja N.T., Titova L.P., Golovatch S.I. 1982. [The myriapod fauna of the Moscow Region] // M.S. Ghilarov (ed.). Pochvennye bespozvonochnye Moskovskoi oblasti. Moscow: Nauka Publ. P.179-200 [in Russian]. 